\title{
Breathing Expression for Intimate Communication Corresponding to the Physical Distance and Contact between Human and Robot
}

\author{
Naoto Yoshida \\ Kansai university \\ Osaka, Japan \\ k463362@kansai-u.ac.jp
}

\author{
Yukari Nakatani ${ }^{\dagger}$ \\ Kansai university \\ Osaka, Japan
}

\author{
Tomoko Yonezawa \\ Kansai university \\ Osaka, Japan \\ yone@kansai-u.ac.jp
}

\begin{abstract}
In this paper, we propose living-being-like breathing expressions concurrent with both aspiration and utterances using a stuffed-toy robot in order to enable intimate interactions. The focus of the research is the impression of the intimacy between the robot and the user corresponding to the physical distance of the two. From the factor analysis of the impression for the word "intimacy" and the distance between the robot and the participants, it is conjectured that the physical intimacy showed strong effects in terms of both warm empathy and tranquility.
\end{abstract}

\section{Categories and Subject Descriptors}

I.2.9 [Robotics]: Commercial robots and applications

\section{General Terms}

Verification

\section{Keywords}

intimate communication, close distance, breathing of robot,close distance

\section{INTRODUCTION}

In recent years, some research studies have explored the idea of building close relationships between humans and robots $[9,7]$.

Similarly, various types of robots have been developed with various forms and sizes that are suitable for various uses and situations such as guidance, sleeping, or outing [11, 3]. In various situations, robots are considered as partners, friends, or family members. In such communication uses, the distance between the robot and the user should be considered as one of the important factors of the communication state, the same as personal space among people.

$\dagger$ currently with Benesse InfoShell Co.,Ltd.
Furthermore, there was a robot that had multiple involuntary expressions on the skin of the robot; namely, goose bumps, sweats, and shivers, like living creatures [10, 8]. In our research, we have also proposed a stuffed-toy robot BRETTER with breathing expression reflecting its utterances for intimate and close communication with physiological and emotive anthropomorphism [5].

The development of these robots with life-like expressions changes the interaction and the definition of familiarity between the human and the robot.

In this paper, using BREAR, our original robot for breathing expression based on physiological state, we verify the factors related to "intimacy" for a robot's expressions based on verification using the two factors of physical distance and contact.

\section{RELATED RESEARCH} \subsection{Intimacy in human-robot communication} Mumm et al. [4] showed the possibility that the distance between the human and the robot affects the behaviors and impression for likability between the user and the robot. Thus there is a possibility of a large factor for intimacy with the robot based on the varying distances between the robot and the user.

On the other hand, some research suggests that physical contact with robots has a positive effect on the users [1, 8]. We expect that physical contact is another factor of intimacy in the human-robot communication.

\subsection{Effects on intimacy of physiology expres- sion}

In recent years, many techniques of emotional expression of a robot have been developed. Nie et al. [6] showed that physical warmth of the hand and handholding increase feelings of friendship and trust toward the robot through a common experience of the robot and the user. Janssen et al. [2] referred to the affective communication using the information of the user's heartbeat. These researches suggest a possibility of affective communication based on biological information.

In this paper, we discuss an expression for intimacy based on the breathing expression of the robot, which is one of the important functions of physiological phenomena in humanrobot communication. 


\section{TEST-BED SYSTEM FOR EXPERIMENT}

Our proposed stuffed-toy robot, which is $25[\mathrm{~cm}]$ high, makes a breathing expression with both air flow and abdominal movement. The robot is covered with a stuffed toy.

Considering the breathing air that corresponds to the robot's utterance in an intimate and close distance, we implemented the breathing mechanism reflecting auditory specification of the robot's voice. The system first analyzes the audio file of the robot's voice to make a simultaneous strength of the outlet flow of the air with the utterance. The strength of the breath is calculated by the high-frequency component, which is considered to make a breathy sound with the outflowing of air.

The audio-signal processing part is implemented in Processing2 ${ }^{*}$, and the results are sent to a microcomputer AVR, Arduino ${ }^{\dagger}$, by serial communication as signals. Finally, the microcomputer changes the breathing mode to the utterance mode, and makes a sigh corresponding to the utterances.

\section{INTIMACY BETWEEN A HUMAN AND A ROBOT THAT BREATHS}

\subsection{Experimental Design}

Purpose of Experiment: We aimed to clarify the factors of "intimacy" between the user and the robot in close communication, in which the user is touching the robot that has the breathing expression. We verified the effect of two factors: the physical distance and physical contact.

Hypotheses: We investigated two independent hypotheses as follows.

1 Physical contact from the user on the robot affects the user's impression of closeness and intimacy.

2 Mutual combination effects between the vocal volume and the distance are as follows:

2-1 Close distance and whisper voice give a positive impression on intimacy,

2-2 Wide distance and whisper voice give negative intimacy,

2-3 Close distance and clear voice give negative intimacy,

And 2-4 wide distance and clear voice give positive intimacy to the participant.

Participants: 23 university students aged from 20 years old to 24 years old (17 males and 6 females).

Conditions: There were eight conditions with three factors: A) voice type, B) contact, and C) distance, as follows:

1 Voice type of the robot (C: Clear, W: Whisper)

\footnotetext{
*Processing2 https://processing.org/

${ }^{\dagger}$ Arduino http://arduino.cc
}

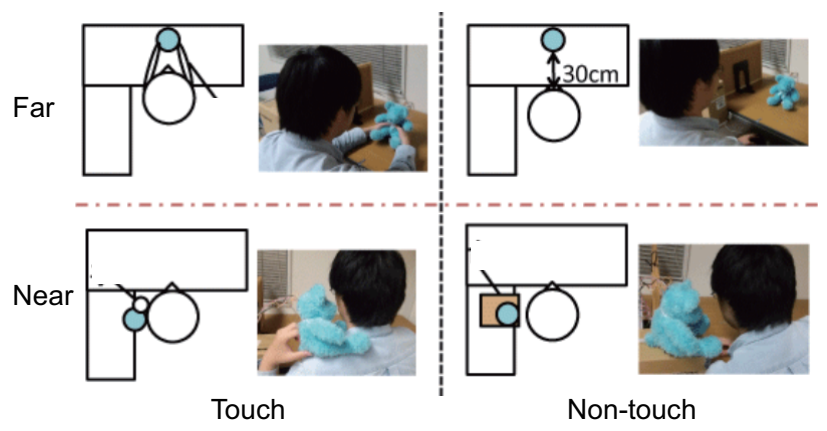

Figure 1: Experimental environments.

2 Physical contact of the user on the robot (T: Touch, n: non-touch)

3 Distance between the robot and the participant $(\mathrm{N}$ : Near, F: Far)

Figure 1 shows the positional settings of the experimental environment. The distance between the participant and the robot is set to be $30[\mathrm{~cm}]$ in the "far" condition. In the condition combining with "close" and "non-touch," the participants were instructed as follows. "Please move your ear close to the robot's mouth." This experiment was prepared as a within-subjects design, so the order of the eight conditions with three factors were randomly arranged for counterbalance.

Procedures and Instructions: The participants were instructed that the experiment is a performance evaluation of the robot.

In each session in the experiment, the participants listened to the voice of the robot for about ten seconds with breathing expressions for each condition. After each session, the experimenter kept the robot and the participant answered the evaluation items by using MOS (means opinion score). The sessions of eight conditions were conducted in random order for counter-balanced evaluation. After the end of all of the conditions, we explained intimacy and closeness, showing pictures of lovers and a crowded train. The participant described their impression of the word "intimacy" for twenty-one adjective pairs using the SD (semantic differential) method. Before the SD method evaluations, the participants could freely ask the definition of the word "intimacy."

Evaluation items for MOS: The participants evaluated the robot in each condition using a five-point scale rating of the relevance (5: very relevant, 4: somewhat relevant, 3 : even, 2: somewhat irrelevant, 1: irrelevant) of the following statements.

1. You experienced a peaceful feeling.

2. You became irritated.

3. You became calm.

4. You felt comforted.

5. The robot seemed to talk to you. 
Table 1: Adjective pairs and factor loadings (Varimax normalized)

\begin{tabular}{|c|r|r|}
\hline Adjective pairs & \multicolumn{2}{|c|}{ Factor loadings } \\
& Factor 1 & Factor2 \\
\hline \hline familiar-alienating & 0.71 & 0.14 \\
\hline friendly-unpeaceful & 0.85 & -0.10 \\
\hline necessary-unnecessary & 0.12 & 0.08 \\
\hline soft-hard & 0.68 & 0.09 \\
\hline leisurely-strained & 0.88 & 0.24 \\
\hline relieved-anxious & 0.75 & 0.14 \\
\hline stimulative-bored & -0.33 & -0.60 \\
\hline relaxed-tensed & 0.85 & 0.19 \\
\hline staid-nervous & 0.46 & 0.56 \\
\hline jolly-broody & 0.63 & 0.08 \\
\hline adult-youthful & -0.51 & 0.12 \\
\hline pure-impure & -0.01 & -0.04 \\
\hline elegant-bestial & -0.22 & 0.13 \\
\hline flat-rough & -0.17 & 0.37 \\
\hline warm-cold & 0.88 & 0.05 \\
\hline poised-restless & 0.03 & 0.96 \\
\hline tranquil-intense & 0.50 & 0.53 \\
\hline simple-complex & 0.18 & 0.26 \\
\hline clean-dirty & 0.44 & 0.14 \\
\hline amiable-unpleasant & 0.84 & 0.21 \\
\hline wet-dried & -0.00 & -0.07 \\
\hline
\end{tabular}

6. The robot seemed as though it is living.

7. The robot should have a mouth like a human.

8. The robot should have muscles to talk.

9. You felt uncomfortable around the robot.

10. The robot wanted to talk with you.

11. You felt you were familiar to the robot.

12. The robot felt familiar to you.

Evaluation items for of the SD method: The participants evaluated the impression for "intimacy" using a five-point scale rating for each adjective pair. The adjective pairs (see Table 1 ) were selected from the research on tactile and romantic relationships. The scores from 1 to 5 are summarized and adopted for the factor analyses.

\subsection{Result of ANOVA for MOS results}

The means and standard deviations are shown in Figure 2 and the results for three factorial ANOVA are shown in Table 2. There are marks corresponding to the significance: + means $p<.10$ and ${ }^{*}$ means $p<.05$. From the results for the evaluation items $\mathrm{Q} 1$ to $\mathrm{Q} 4$, which are verifying the familiarity of the participants toward the robot, there were significances based on the factor of voice type; while there is a significant result in Q3 based on the factor of touch. From the results for Q5 to Q8, that are related to the impression of living beings, there were significant results in terms of the factor of touch. The results for Q9 to Q12 are the evaluations of intimacy. There was significance in Q9 in the factor of closeness.

\subsection{Extraction of Factors for the impression of the word "intimacy"}

The factor analysis was performed on the results of SD method ratings for the twenty-one adjective pairs. According to the difference in eigenvalues by a major-factor method
Table 2: Results for three factorial ANOVA.

\begin{tabular}{cccccccc}
\hline & $F(A: C W)$ & $p(C W)$ & $F(B: T n)$ & $p(T n)$ & $F(C: N F)$ & $p(N F)$ & Interaction \\
\hline 1 & 27.1 & $<0.01^{* *}$ & 0.16 & 0.68 & 0.35 & 0.56 & - \\
2 & 18.1 & $<0.00^{* *}$ & 0.00 & 1.00 & 0.00 & 1.00 & - \\
3 & 23.9 & $<0.01^{* *}$ & 4.56 & $0.04^{*}$ & 3.37 & $0.07+$ & - \\
4 & 21.0 & $<0.00^{* *}$ & 2.00 & 0.17 & 1.07 & 0.31 & - \\
5 & 14.7 & $<0.01^{* *}$ & 4.21 & $0.05+$ & 2.63 & 0.11 & $\mathrm{~A}\left((\mathrm{~b} 2)<0.01^{* *}, \mathrm{~B}(\mathrm{a} 2)<0.01^{* *}\right.$ \\
6 & 2.60 & 0.12 & 17.5 & $<0.01^{* *}$ & 2.08 & 0.16 & - \\
7 & 3.41 & $0.07+$ & 14.4 & $<0.01^{* *}$ & 2.50 & 0.12 & $\mathrm{AC}+$ \\
8 & 5.84 & $0.02^{*}$ & 11.7 & $<0.01^{* *}$ & 0.11 & 0.74 & - \\
9 & 33.5 & $<0.00^{* *}$ & 0.04 & 0.83 & 11.7 & $<0.01^{* *}$ & $\mathrm{C}(\mathrm{b} 1)<0.01^{* *}$ \\
10 & 4.92 & $0.03^{*}$ & 7.34 & $0.01^{*}$ & 1.27 & 0.27 & $\mathrm{AC}+$ \\
11 & 35.6 & $<0.00^{* *}$ & 10.7 & $<0.01^{* *}$ & 0.25 & 0.61 & - \\
12 & 28.6 & $<0.01^{* *}$ & 9.62 & $<0.01^{* *}$ & 0.24 & 0.62 & - \\
\hline
\end{tabular}

as shown in Table 1, we considered two factors from the contribution ratios. There are underlines on the adjective pairs with the factor loading more than 0.5 or less than -0.5 . The first and second factors are considered from the results of the eigenvalues. The factor matrix was rotated by a Varimax method.

The first factor was named the "Friendly" factor because the eigenvalue of 'familiar-alienating' and 'friendly-unpeaceful' were high. The second one was named the "Calmness" factor because the eigenvalue of 'poised-restless' and 'stimulativebored' were high.

\section{DISCUSSION}

\subsection{Intimacy with breathing robot}

We first discuss the impression for "intimacy" of the breathing robot. As shown in the results of the factor analyses, there were two factors; the first one was the "Friendly" factor and the second one was the "Calmness" factor. The first factor is considered to lead to direct closeness. Unexpectedly, there was an aspect of the stimulating (or jittering) versus stabilized (calm or bored) factor in the impression of "intimacy." It is conjectured that the difference between lover-like or family-like intimate situation causes the second factor. From the viewpoint as above, the artificial presence such as our proposed robot should behave corresponding to the detailed settings of the relationship.

\subsection{Effect of physical distance and contact on intimate communication}

Next we discuss the significant results in ANOVA for MOS results. First, related to the hypothesis 1), there were significant results by the factor of touch in direct or indirect items for familiar impressions. Thus it is conjectured that the physical contact with the stuffed-toy robot makes the user familiar to the robot.

Related to the hypothesis 2) on the living-being-like expressions, the condition of breathing with touch is considered in two cases: perceived by abdominal motion or breathy air at a close distance. From the results for Q5 to Q8, there were significant results for the factor of touch while there was not any significance in terms of the distance. It is conjectured that the internal mechanism such as abdominal motion is observed by touching. 


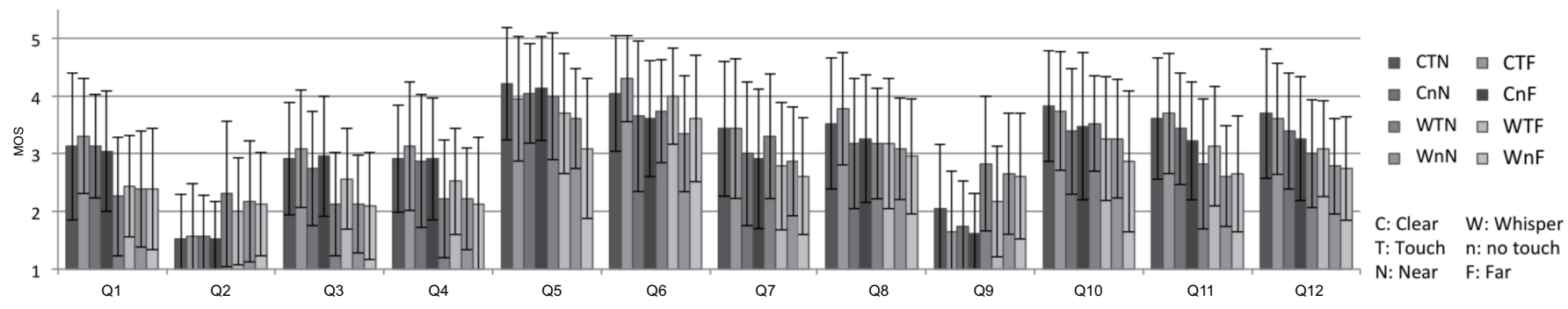

Figure 2: Results of subjective evaluation (MOS).

On the hypothesis 2-1), in the configuration of the evaluation, the effect of the voice quality seemed to affect the evaluation from the significant results by the voice factor. The negative results of the living-being-like expression could be changed to positive if the voice becomes natural and the premised relationship is given as a familiar one.

\section{CONCLUSION}

In this paper, we have introduced a robot with breathing expressions corresponding to its utterances and breath. Focusing on the change of the impression for intimacy corresponding to the relational position, between the robot and the user, that is the distance far or close, we have evaluated the effectiveness of our proposed system. Through the results of MOS, it was conjectured that the close-distance communication provides some uncomfortable situations through the too-vivid expressions that make it seem real. From the factor analyses of SD, the impression for intimacy is affected by the "Friendly" factor and the "Calmness" factor. The lively expressions of the robot in close distance seems to make negative impressions on the "Friendly" factor. As for future work, it is necessary to evaluate the breathing expression of the robot at a close distance in order to evaluate the relationship, especially intimacy, between the robot and the user.

\section{ACKNOWLEDGMENTS}

This research was supported in part by JSPS KAKENHI 15H01698, JSPS KAKENHI 25700021, and JSPS KAKENHI 24300047. The authors would like to thank the participants in the experiment.

\section{REFERENCES}

[1] C. DiSalvo, F. Gemperle, J. Forlizzi, and E. Montgomery. The hug: an exploration of robotic form for intimate communication. In Robot and Human Interactive Communication, 2003. Proceedings. ROMAN 2003. The 12th IEEE International Workshop on, pages 403-408. IEEE, 2003.

[2] J. H. Janssen, J. N. Bailenson, W. Ijsselsteijn, J. H. Westerink, et al. Intimate heartbeats: Opportunities for affective communication technology. Affective Computing, IEEE Transactions on, 1(2):72-80, 2010.

[3] T. Kashiwabara, H. Osawa, K. Shinozawa, and M. Imai. Teroos: a wearable avatar to enhance joint activities. In Proceedings of the SIGCHI Conference on Human Factors in Computing Systems, pages 2001-2004. ACM, 2012.
[4] J. Mumm and B. Mutlu. Human-robot proxemics: Physical and psychological distancing in human-robot interaction. In Proceedings of the 6th International Conference on Human-robot Interaction, HRI '11, pages 331-338, New York, NY, USA, 2011. ACM.

[5] Y. Nakatani and T. Yonezawa. Breatter: a simulation of living presence with breath that corresponds to utterances. In Proceedings of the 2014 ACM/IEEE international conference on Human-robot interaction, pages 254-255. ACM, 2014.

[6] J. Nie, M. Park, A. L. Marin, and S. S. Sundar. Can you hold my hand? physical warmth in human-robot interaction. In Human-Robot Interaction (HRI), 2012 7th ACM/IEEE International Conference on, pages 201-202. IEEE, 2012.

[7] Y. Yamaji, T. Miyake, Y. Yoshiike, P. R. De Silva, and M. Okada. Stb: human-dependent sociable trash box. In Human-Robot Interaction (HRI), 2010 5th ACM/IEEE International Conference on, pages 197-198. IEEE, 2010.

[8] S. Yanaka, M. Ishida, T. Kosaka, M. Hattori, and H. Sato. Resolution of sleep deprivation problems using zzzoo pillows. In Proceedings of the Virtual Reality International Conference: Laval Virtual, page 25. ACM, 2013.

[9] S. Yohanan and K. E. MacLean. The role of affective touch in human-robot interaction: Human intent and expectations in touching the haptic creature. International Journal of Social Robotics, 4(2):163-180, 2012.

[10] T. Yonezawa, X. Meng, N. Yoshida, and Y. Nakatani. Involuntary expression of embodied robot adopting goose bumps. In Proceedings of the 2014 ACM/IEEE international conference on Human-robot interaction, pages 322-323. ACM, 2014.

[11] T. Yonezawa and H. Yamazoe. Wearable partner agent with anthropomorphic physical contact with awareness of user's clothing and posture. In Proceedings of the 2013 International Symposium on Wearable Computers, ISWC '13, pages 77-80, New York, NY, USA, 2013. ACM. 